\title{
His Bundle Pacing Improves LV Diastolic Function in Patients with Heart Failure with Preserved Systolic Function
}

\author{
Bengt Herweg ${ }^{1}$, Dipayon Roy ${ }^{1}$, Allan Welter-Frost ${ }^{1}$, Cody Williams ${ }^{1}$, Arzu Ilercil ${ }^{1}$, and \\ Pugahendhi Vijayaraman ${ }^{2}$ \\ ${ }^{1}$ University of South Florida \\ ${ }^{2}$ Geisinger Health
}

November 1, 2021

\begin{abstract}
Cardiac resynchronization therapy (CRT) is highly effective for patients with left bundle branch block (LBBB), heart failure and left ventricular (LV) systolic dysfunction. Chronic right ventricular (RV) apical pacing is associated with pacing-induced cardiomyopathy and can be associated with exertional intolerance. The goal of this study was to assess the acute hemodynamic effects of His-bundle Pacing (HBP) compared to RV apical pacing in absence of LV systolic dysfunction in patients with exertional intolerance. Our patient population consisted of 5 patients with preserved LV systolic function and complete AV block. All five patients suffered from exertional intolerance in spite of preserved LV systolic function. At the time of generator change, all patients underwent implantation of a HBP lead. The QRS duration decreased from $179 \pm 13 \mathrm{~ms}$ with RVP to $113 \pm 6$ with HBP $(\mathrm{p}<0.001)$. Compared to RVP, HBP was associated with significantly longer diastolic filling time and improved septal early diastolic myocardial relaxation velocity ( $\left.E^{\prime}\right)$. Four of five patients noted acutely improved exertional dyspnea. In patients with AV block, exertional intolerance and preserved LV systolic function who are treated with chronic RV apical pacing, HBP may improve acute diastolic function and symptoms of exertional intolerance when compared to RV apical pacing. Randomized controlled trials are warranted to explore the effects of conduction system pacing in this unique patient population.
\end{abstract}

\section{Hosted file}

Diastolic_HBP_Manuscript_S2.docx available at https://authorea.com/users/443916/articles/ 543792-his-bundle-pacing-improves-lv-diastolic-function-in-patients-with-heart-failurewith-preserved-systolic-function 